\title{
OS DIAGNÓSTICOS DE ENFERMAGEM DA TAXONOMIA DA NANDA EM MULHERES COM O FILHO PREMATURO HOSPITALIZADO E O SISTEMA CONCEITUAL DE KING*
}

\author{
Cláudia Silveira Viera** \\ Lídia Aparecida Rossi***
}

\begin{abstract}
VIERA, C.S.; ROSSI, L. Os diagnósticos de enfermagem da taxonomia da NANDA em mulheres com o filho prematuro hospitalizado e o sistema conceitual de King. Rev.latino-am.enfermagem, Ribeirão Preto, v. 8, n. 6, p. 110-116, dezembro 2000.
\end{abstract}

Os objetivos deste estudo foram identificar os diagnósticos de enfermagem em mães com o filho prematuro hospitalizado em UTI Neonatal, mediante a utilização do Sistema Conceitual de King e da Taxonomia I dos diagnósticos de enfermagem da NANDA (1999). Para tanto utilizou-se a metodologia de estudo de caso aplicada a 35 puérperas. Foram identificados oito diagnósticos de enfermagem com freqüência igual ou maior que 50\%, a saber, Risco para Infecção, Risco para Injúria, Integridade Tissular Prejudicada, Manutenção da Saúde Alterada, Risco para Amamentação Ineficaz; Risco para Alteração no Desenvolvimento do Apego entre Pais e Filho; Medo; Conflito no Desempenho de Papéis dos Pais.

UNITERMOS: diagnóstico de enfermagem, recém-nascido prematuro, puerpério

\section{INTRODUÇÃO}

Para as mulheres, o processo de parturição faz parte de suas vidas integrado a outros aspectos, principalmente porque é mediante esse processo que passam a adquirir um outro papel perante o grupo social do qual estão inseridas, o de ser mãe.

O nascimento de um filho, promove uma reestruturação da família para receber esse novo ser e cria uma expectativa para aqueles que ficam do outro lado, esperando ansiosamente para conhecer o recém-chegado. $\mathrm{O}$ que acontecerá com essa família se, porventura, o recémnascido não puder ficar com a mãe no Alojamento Conjunto ou, ainda pior, se ele permanecer hospitalizado? Muitos sentimentos poderão surgir, prejudicando tanto a relação familiar, como a dos pais e com o recém-nascido, devido a essa separação inesperada. Como esses problemas podem ser detectados pelos profissionais que cuidam das mães e de seus filhos recém-nascidos?

O nascimento de um filho antes do tempo, ou com complicações patológicas que implicam em hospitalização, é uma situação difícil e penosa para todos na família e, principalmente, para a mãe que, muitas vezes, sente-se culpada por esse acontecimento.

Acredita-se que quando a assistência é planejada, levantando dados, identificando os problemas de forma individualizada, ou seja, considerando cada família como um núcleo especial, é possível que o enfermeiro se instrumentalize para auxiliar essa família a transpor esse momento de crise. Assim, a família pode se sentir como parte desse mundo onde está seu filho e ser motivada a participar dele.

Para KING (1981) os seres humanos em interação com o meio ambiente são o foco da enfermagem, cuja atribuição seria a de conduzi-los a um estado de saúde, ou seja, a estarem aptos para atuar em seus papéis sociais.

KING (1981) desenvolveu, através de sua teoria sobre o Sistema Conceitual, quatro conceitos centrais, sendo eles: o meio ambiente, a saúde, o ser humano e a enfermagem. O Sistema Conceitual é composto por três sistemas interatuantes: o sistema pessoal, no qual insere a idéia de que cada ser humano forma um sistema, e de que por meio de interações, esses seres humanos formarão duplas, pequenos ou grandes grupos, constituindo assim um outro sistema, ao qual denomina de sistema interpessoal. Esses seres humanos agrupados, estruturarão organizações de acordo com suas necessidades e interesses, formando um novo sistema, o

\footnotetext{
* Este trabalho constitui parte de Dissertação de Mestrado apresentada ao Programa - Convênio Mestrado Interinstucional - PósGraduação Área de Enfermagem Fundamental do Departamento de Enfermagem Geral e Especializada da Escola de Enfermagem de Ribeirão Preto da Universidade de São Paulo e Departamento de Enfermagem da Universidade do Oeste do Paraná - Cascavel ** Enfermeira. Docente junto ao Departamento de Enfermagem da Universidade do Oeste do Paraná - Cascavel

*** Enfermeira. Professor Doutor do Departamento de Enfermagem Geral e Especializada da Escola de Enfermagem de Ribeirão Preto da Universidade de São Paulo. Endereço: Av. Bandeirantes, 3900 - Monte Alegre - 14040-902 - Ribeirão Preto - São Paulo - Brasil. E-mail: rizzardo@glete.eerp.usp.br
} 
sistema social (GEORGE, 1990). Assim, o Sistema Conceitual da teoria proposta por KING (1981) se baseia nesses três sistemas interatuantes e dinâmicos, identificando conceitos relevantes dentro de cada um deles.

Com base nos conceitos propostos por KING (1981), parte-se do pressuposto, neste estudo, de que a presença da mãe contribui para o restabelecimento da criança doente, que ela se recupera mais rapidamente se estiver sob os cuidados da mãe e que a separação mãe e filho compromete a formação do vínculo afetivo entre eles. Percebe-se então que a identificação dos diagnósticos de enfermagem da mãe do recém-nascido $(\mathrm{RN})$ hospitalizado, considerando a família como participante deste momento, possibilitará a organização da assistência de enfermagem com o objetivo de propiciar condições à essa mãe para suportar a situação vivida de forma que possa contribuir positivamente para a promoção, restauração e manutenção da saúde de seu filho, bem como a resolução das necessidades da mãe evidenciadas durante esse período.

KING (1981) contribui para esse entendimento quando afirma que o homem é um sistema semi-aberto, em interação constante com o meio ambiente, não podendo ser assistido individualmente sem envolver sua família nesse cuidado. Para essa autora, o sistema pessoal influencia e é influenciado pelo sistema interpessoal e pelo social. Assim, crê-se que os diagnósticos de enfermagem que envolvem as mães dos RNs prematuros hospitalizados são influenciados por essa situação de crise tanto individualmente quanto nas relações familiares. A presença da mãe junto do $\mathrm{RN}$ e a identificação de suas necessidades poderá contribuir para a melhor interação mãe e filho e melhora clínica do RN.

Muitos estudos foram realizados com o objetivo de levantar como a hospitalização dos recém nascidos é enfrentada pelos pais e qual o seu significado (MILES et al., 1992; GOMES, 1992; BEZERRA \& FRAGA, 1996; SOUZA, 1997). Outros enfocaram a família e suas percepções sobre a hospitalização de seus recém-nascido e/ou crianças (ANGELO, 1982; 1997; MOTTA, 1998); outros trabalharam a percepção da família frente a chegada de um novo membro e também os cuidados dessa família e/ou pais para com o RN (BOECHS, 1992, SCOCHI et al., 1992). GARCIA et al. (1993) construíram um instrumento de coleta de dados com base no Sistema Conceitual de King (1981) e aplicaram-no a uma gestante adolescente, visando testar a aplicabilidade desse modelo a essa clientela. MARQUES \& ELSEN (1994) estudaram a utilização do Sistema Conceitual e a Teoria do Alcance de Metas de King (1981) na assistência de enfermagem a parturientes. Tem-se ainda relatos sobre as intervenções e/ou opiniões de profissionais de enfermagem frente a família e ou pais diante da hospitalização de seus filhos como os realizados por BARBOSA (1990); KIMURA (1996) e HAMELIN et al. (1997), mas poucos abordaram os diagnóstico de enfermagem apresentados pela mãe no pósparto. Neste último aspecto, pode-se citar os estudos de CHU
LY (1997); WILLIANS \& COOPER (1993); TRIBOTI et al. (1988) e STEVENS (1988). Entretanto, não encontrou-se estudos que enfocassem os diagnósticos de enfermagem das mães que tiveram a vivencia de ter seus filhos prematuros hospitalizados.

Com base no referencial teórico do Sistema Conceitual de KING (1981), o qual norteou o desenvolvimento do estudo e mediante a utilização da Taxonomia da North American Nursing Diagnoses Association NANDA (1999), elaborou-se através do processo de raciocínio diagnóstico proposto por RISNER (1990) os diagnósticos de enfermagem para essa clientela. Compreendese que o estabelecimento das afirmativas diagnósticas ocorre na segunda etapa do processo de enfermagem. Cabe ressaltar que esse processo é composto por fases, a saber, coleta de dados, diagnóstico de enfermagem, planejamento, implementação e avaliação. Neste estudo, optou-se por descrever as duas primeiras fases do processo de enfermagem, embora após a identificação dos diagnósticos de enfermagem as intervenções necessárias tenham sido realizadas.

Assim, o objetivo deste estudo foi identificar os diagnósticos de enfermagem apresentados pelas mães que tiveram seu filho prematuro hospitalizado em uma UTI Neonatal.

\section{METODOLOGIA}

Considerando o objetivo deste estudo e a especificidade da clientela estudada, optou-se pela metodologia do estudo de caso conforme descrita por LÜDKE \& ANDRÉ (1986). A pesquisa foi desenvolvida no Hospital Regional de Cascavel que é uma instituição pública e estadual, que conta com cento e oitenta leitos, dos quais praticamente $90 \%$ são ocupados por pacientes do Sistema Único de Saúde (SUS). Do total de leitos, nove são destinados para o Centro Obstétrico, 16 para o sistema de Alojamento Conjunto Obstétrico e 14 para Unidade de Terapia Intensiva Neonatal. O Centro Obstétrico desta instituição realiza em média, 309 partos mensais. Na Unidade de Terapia Intensiva Neonatal há uma média de 10,6 internações ao mês devido a prematuridade.

Os dados foram coletados na unidade de internação do Alojamento Conjunto Obstétrico dessa instituição, onde, mediante a interação com a equipe de enfermagem da unidade, obteve-se informações referentes a hospitalização no local de puérperas com RNs prematuros e hospitalizados. De posse dessas informações, partiu-se para o contato com a puépera, explanando sobre a pesquisa, sendo garantido o anonimato às pacientes participantes do estudo e questionando-as sobre seu interesse de participar deste. Fizeram parte da amostra deste estudo, as puérperas de qualquer idade que tiveram seu filho prematuro internado na UTI Neonatal e que estavam ainda hospitalizadas, ou seja, nas primeiras 72 horas após o 
parto. Essas puérperas foram reavaliadas durante o período de hospitalização de seu filho, momento este em que se questionou acerca de dados como rotina familiar e observouse a visita da mãe junto ao RN.

Os dados foram coletados pela primeira autora deste estudo, durante um período de quatro meses, maio a agosto de 1999, consistindo da entrevista e exame físico das mães que estavam na situação descrita e que se propuseram a participar do estudo, mediante a assinatura de um consentimento durante o primeiro contato com a primeira autora deste estudo, sendo que àquelas menores de 21 anos solicitou-se também a assinatura de um responsável.

Utilizou-se como roteiro de coleta e registro dos dados um instrumento elaborado previamente, compondo-se de entrevista e exame físico, construído com base no Sistema Conceitual proposto por Imogene King (1981), no qual as proposições estão em consonância com os objetivos deste estudo, pois engloba o indivíduo no seu contexto social, quando fala do sistema pessoal, interpessoal e social.

Após a elaboração da primeira versão do instrumento, essa foi submetida a um pré-teste segundo POLIT \& HUNGLER (1995). Como recomendam essas autoras, o instrumento foi submetido à apreciação de cinco enfermeiros docentes da área de Materno Infantil da EERP-USP e das Universidades Estadual do Oeste do Paraná e Federal da Paraíba e de uma enfermeira atuante na área de Centro Obstétrico e Alojamento Conjunto Obstétrico do Hospital Regional de Cascavel. Foram incorporadas no instrumento as sugestões propostas pelos enfermeiros no pré-teste.

Depois da aprovação do estudo por um Comitê de Ética e Pesquisa, realizou-se ainda um estudo piloto conforme proposto por POLIT \& HUNGLER (1995). Assim, o instrumento foi aplicado a três puérperas que tiveram seus filhos prematuros hospitalizados, cujos dados obtidos não foram incluídos nos resultados do estudo.

Durante todo o processo de coleta de dados, as informações subjetivas e as impressões do pesquisador foram confirmados com as puérperas e, durante o encontro seguinte, eram discutidos os problemas percebidos por ambos.

Em um segundo momento do estudo, estabeleceuse os diagnósticos de enfermagem com base na Taxonomia I dos Diagnósticos de Enfermagem da NANDA (1999), utilizando-se o processo de raciocínio diagnóstico proposto por RISNER (1990), de acordo com a adaptação de CARVALHO \& JESUS (1997). Realizada essa etapa, a lista de diagnósticos e seu respectivo processo de raciocínio foram entregues a duas pessoas, sendo uma delas a segunda autora deste estudo e a outra docente da área obstétrica, para que fossem ou não confirmados os diagnósticos.

Considera-se importante ressaltar em que na medida que foram estabelecidos os diagnósticos de enfermagem, as pacientes receberam a assistência de enfermagem específica, embora as intervenções de enfermagem não constituíssem foco de interesse deste estudo.

\section{RESULTADOS E DISCUSSÃO}

O maior número de puérperas estudadas tinha entre 21 a 25 anos, correspondendo a 11 puérperas $(31,42 \%)$ e entre 31 a 35 anos, correspondendo a 10 puérperas (28,57\%); 23 $(65,14 \%)$ não tinham vínculo empregatício, não contribuindo para a renda familiar, a qual girou em torno de um a três salários mínimos para 19 puérperas (54,28\%). Pode-se dizer que os afazeres da casa, o cuidado com os outros filhos e a baixa renda familiar poderiam implicar em um menor número de visitas ao RN hospitalizado, aumentando assim a separação mãe e RN e dificultando a formação dos laços de apego, ou ainda acarretar alterações na rotina diária dessas famílias, levando também ao estresse devido a preocupação com a situação.

A maior incidência da amostra foi de mulheres primíparas, compreendendo 12 do total de puérperas estudadas $(34,28 \%)$. Esse dado implica na caracterização das puérperas como inexperientes e vivenciando uma situação nova que é a de ser mãe. Percebeu-se que 26 puérperas, $74,28 \%$ da amostra estudada, realizaram consultas pré-natal, duas (5,71\%) não realizaram consultas pré-natal. Foi observado também que sete puérperas $(20 \%)$ iniciaram tardiamente o pré-natal e justificaram essa situação ou por não saberem que estavam grávidas ou porque residiam distante da unidade básica.

As complicações obstétricas de maior incidência neste estudo foram a rotura precoce das membranas ovulares, ocorrendo em 10 puérperas (28,57\%) e a Doença Hipertensiva Específica da Gestação em cinco puérperas (14,28\%) dentre o total estudado. A idade gestacional das mulheres estudadas variou de 26 a 36 semanas.

Foram identificados 18 diagnósticos de enfermagem a saber, Risco para Infecção, Integridade Tissular prejudicada, Manutenção da Saúde Alterada, Risco para Amamentação Ineficaz, Risco para Alteração no Desenvolvimento do Apego entre Pais e Filho, Medo, Estratégias de Ineficazes de Resolução Individual, Excesso de Volume de Líquido, Constipação Colônica, Dor, Distúrbio na Imagem Corporal, Risco para Injúria, Conflito no Desempenho de Papéis dos Pais, Nutrição Alterada: Ingestão Menor do que as Necessidades Corporais, Processos Familiares Alterados: alcoolismo, Manutenção do Lar Prejudicada, Mucosa Oral Alterada e Interação Social Prejudicada.

Classificou-se os diagnósticos de enfermagem com freqüência igual ou maior que 50\% no Sistema Pessoal como: Risco para Infecção, Manutenção da Saúde Alterada, Risco para Injúria, Integridade Tissular Prejudicada e no Sistema Interpessoal como: Risco para amamentação Ineficaz, Medo, Risco para Alteração no Desenvolvimento do Apego entre Pais e Filho e Conflito no Desempenho de Papéis dos Pais. Já no Sistema Social obteve-se apenas um diagnóstico, Interação Social Prejudicada, o qual foi apresentado por duas puérperas $(5,71 \%)$. O fato de que apenas um diagnóstico de enfermagem 
foi classificado nesse sistema pode ser explicado pelo enfoque da Taxonomia de Diagnósticos de Enfermagem da NANDA (1999) ainda ser eminentemente biológico, com poucos diagnósticos no padrão de resposta humana relacionar. Um outro fato que contribui para essa situação pode ser a nossa própria cultura que valoriza pouco os aspectos sociais do cuidado.

Tal situação foi surpreendente, pois na apresentação dos diagnósticos de enfermagem detectados, percebeu-se a maior incidência de diagnósticos biológicos e que se referiam a puérpera, porém quando esta classificação foi feita, podese observar que os diagnósticos de enfermagem encontraramse distribuídos igualmente entre o sistema pessoal e interpessoal. Acredita-se que este resultado seja decorrente do envolvimento desse sistema, que esteve claramente presente na situação vivenciada mediante a relação existente entre a puérpera e o restante de sua família, ou seja, o RN, o marido, os outros filhos. Dessa forma, cabe salientar que considerando o indivíduo como um todo, que interage com os outros e com o meio social, seria improvável que não se fizessem presentes os diagnósticos de enfermagem nas dimensões enquanto Sistema Interpessoal e Social. É importante ressaltar que procuramos destacar igualmente os três sistemas nos tópicos que compõem o instrumento de coleta de dados, bem como na sua própria utilização no momento em que os dados foram coletados.

Entre os diagnósticos que apresentaram freqüência igual ou maior que $50 \%$, destaca-se o Risco para Amamentação Ineficaz identificado em 35 das puérperas estudadas (100\%). Esse diagnóstico não é apresentado pela Taxonomia I dos diagnósticos de enfermagem (1999), entretanto, com base no estudo de VALE (1999), que propõe a inclusão deste diagnóstico na Taxonomia da NANDA e, nos fatores de risco identificados na amostra estudada, optouse pela sua confirmação considerando também a definição proposta por essa autora: estado em que a mãe ou bebê apresentam maior vulnerabilidade para dificuldade na amamentação. Acredita-se que neste estudo, este seja um diagnóstico de risco, pela situação vivenciada, pois como ainda não se estabeleceu a amamentação, não se pode avaliar sua eficácia e sim os fatores de risco que predispõem essa mulher a não estabelecer a amamentação. Uma vez detectados os fatores de risco para este acontecimento, pode-se trabalhar no sentido de evitar que isto ocorra.

A identificação desse diagnóstico deu-se mediante a constatação de fatores de risco como a prematuridade do $\mathrm{RN}$, que leva a hospitalização, propiciando a separação do RN de sua mãe e família e a oportunidade insuficiente de amamentação ao seio, levando também a uma inconstância da sucção do seio materno. Nesse fator, pode-se incluir o número reduzido de visitas da mãe ao $\mathrm{RN}$, as condições clínicas do RN e o uso de alimentação artificial. Associado a esses fatores de risco tem-se o desconhecimento pelas puérperas de formas de manutenção da lactação, como a extração manual de leite das mamas com freqüência, a ingesta hídrica adequada durante a hospitalização do $\mathrm{RN}$, traduzindose no fator de risco de déficit de conhecimento sobre manutenção da lactação.

Outros fatores de risco que emergiram neste diagnóstico foram o medo materno, referente a possibilidade de secar o leite e a preocupação com o RN, a história prévia de fracasso na amamentação, relatada como "secar" o leite, doença da mãe e tipo de mamilo, e a presença de anomalia do seio materno (mamilo invertido ou semi-invertido).

Cabe salientar que o diagnóstico de Risco para Vinculação Pais-Filho Alterada, conforme a tradução apresentada no livro "Manual de Diagnóstico de Enfermagem”, edição em português, CARPENITO (1999) teve seu rótulo alterado nesse estudo para Risco para Alteração no Desenvolvimento do Apego entre Pais e Filho, por considerar a palavra apego mais usual em literaturas sobre o tema (KLAUS \& KENNELL, 1993). Na identificação desse diagnóstico foram encontrados os fatores de riscos, a saber, $a$ hospitalização da criança desfavorecendo um contato efetivo entre os pais e essa, podendo alterar a organização do comportamento, à dificuldade de adiar a própria gratificação em beneficio do bebê (mãe e adolescente) acarretando a dificuldade de estabelecer apego mãe e filho e a separação devido a hospitalização do RN pela prematuridade. Assim, tem-se que o nascimento prematuro do RN acarreta uma hospitalização, muitas vezes prolongada, a qual propicia uma separação do RN de sua mãe e família podendo ocasionar uma dificuldade no estabelecimento do apego. Segundo BRAZELTON (1988); KLAUS \& KENNELL (1993), o desenvolvimento do apego teria início desde a gestação e seria aprimorado com o nascimento e interação mãe e filho. A separação impossibilita o estabelecimento de uma relação de interação entre a mãe, o filho e o pai através da qual se firmaria um vínculo inicial para dar continuidade a formação do apego. KLAUS \& KENNELL (1993) definem o apego como um relacionamento impar entre duas pessoas, específico e duradouro ao longo do tempo, que começa a existir a partir do momento em que se decide ter um filho, passando a se desenvolver na gestação e se intensificando após o parto. A esse momento de relacionamento entre duas pessoas KING (1981) denomina interação.

Das 35 puérperas que apresentaram esse diagnóstico apenas uma teve como fator de risco o fato de ser adolescente e ter dificuldade de enfrentar essa situação associada ao processo de adolescer. Da mesma forma esses fatores associados a prematuridade do $\mathrm{RN}$, ao déficit de conhecimento sobre as práticas de amamentação estão relacionados ao Risco para Amamentação Ineficaz.

Observou-se a falta de instrução adequada sobre práticas de saúde em 32 puérperas dentre o total estudado (91,42\%), situação que está associada a Manutenção da Saúde Alterada. Como característica definidora do diagnóstico de Manutenção da Saúde Alterada observa-se a demonstração 
do desconhecimento sobre as práticas de saúde no que se refere aos exames preventivos de câncer e planejamento familiar. Essas puérperas relataram ainda, que quando apresentam problemas de saúde procuram farmácias e pessoas consideradas mais experientes entre os seus familiares.

O Conflito de Desempenho de Papéis dos Pais, expressou a preocupação com o desempenho de papéis no que se refere a mudança na vida e funcionamento familiar. Foram identificados como fatores relacionados para esse diagnóstico nesse estudo a separação da criança secundária à: hospitalização (pela prematuridade), adolescência, pais solteiros, interrupção da vida familiar secundária à: visitas freqüentes ao hospital e interrupção da vida familiar secundária à: adição de um novo membro à família (o RN). Como característica definidora foi observada a preocupação expressa pela mãe com mudanças no papel materno, no funcionamento familiar. Nesse sentido, as puérperas avaliadas relataram fatos como preocupação com outros filhos e/ou membros da família, com a condição financeira, com a presença de mais um membro na família, relataram ainda a preocupação com as mudanças na rotina diária de casa e com mudanças nos papéis dos componentes da família.

O diagnóstico de Medo foi caracterizado mediante a demonstração pelas puérperas da capacidade de identificar as razões desse sentimento. Nesse sentido, o objeto do medo identificado pelas puérperas se refere a incerteza do que pode acontecer com o RN hospitalizado. MILES et al. (1992); ZAVASCHI et al. (1985) e BOWLBY (1981) relataram os sentimentos de medo, apreensão e culpa como decorrentes do temor de terem feito algo errado que levasse a prematuridade e ainda frustração frente ao nascimento de um $\mathrm{RN}$, que não é nada semelhante ao filho idealizado durante a gestação e, ansiedade quanto a possibilidade de perder seus filhos.

Assim, pode-se dizer que todos esses sentimentos são fatores que contribuem para o diagnóstico de enfermagem de Medo encontrado neste estudo, em que se detectou como fator relacionado para esse diagnóstico a separação da criança de sua família, decorrente da necessidade de hospitalização pela prematuridade e como características definidoras a habilidade para identificar o objeto do medo, ou seja, relato de medo e preocupação com o que pode acontecer ao $\mathrm{RN}$ e, ainda, a manifestação de sentimentos de apreensão (choro, tristeza) e de culpa (relato de que sente remorso).

O Risco para Injúria, identificado em 20 puérperas $(57,14 \%)$, teve como fator de risco mais freqüente o perfil anormal do sangue apresentado por 12 dessas puérperas $(60 \%)$. Assim, um fator de risco presente nesse diagnóstico é a diminuição de hemoglobina, que pode acarretar menor oxigenação cerebral e vertigens. As mudanças fisiológicas, as quais as mulheres estão sujeitas no pós parto, também indicam a presença desse diagnóstico. Essas alterações promovem, nas primeiras 48 horas pós parto, modificações na volemia levando a um retorno venoso mais lento (REEDER et al., 1997).
Odiagnóstico de enfermagem de Risco para Infecção foi estabelecido com base na presença dos seguintes fatores de risco: procedimentos invasivos (procedimento cirúrgico, venopunções, sondagem vesical); defesa primária insuficiente: solução de continuidade da pele (presença de algum tipo de solução de continuidade da pele como laceração do períneo, cicatriz cirúrgica - episiorrafia ou cesariana); defesa secundária insuficiente (presença de hemoglobina ou hematócrito diminuídos) e rotura precoce das membranas ovulares. O fator de risco, defesa primária insuficiente, neste caso a presença de solução de continuidade da pele, se fez presente em 32 do total de puérperas estudadas $(91,42 \%)$, dessa forma, pode-se dizer que o diagnóstico de enfermagem de Integridade Tissular Prejudicada, o qual foi apresentado por 32 puérperas do total estudado $(91,42 \%)$ está incluso no diagnóstico de Risco para Infecção. Portanto, considera-se o Risco para Infecção como o diagnóstico mais amplo e, desse modo, foi mantido como diagnóstico de enfermagem definitivo. Assim, entende-se que as metas e objetivos traçados com base nesse diagnóstico, indicam intervenções para o diagnóstico de enfermagem de Integridade Tissular Prejudicada.

Percebeu-se também que a separação da díade mãe e filho, provocada pela hospitalização do RN, está associada a mais de um diagnóstico (Medo, Conflito de Desempenho de Papéis dos Pais, Risco para Amamentação, Risco para alteração no desenvolvimento do Apego).

CARPENITO (1999) cita alguns desses diagnósticos encontrados, como o Risco para Infecção e Manutenção da Saúde Alterada, classificando-os como diagnósticos de enfermagem do período de pós-parto em geral.

\section{CONSIDERAÇÕES FINAIS}

Pode-se perceber que a experiência de se ter um filho prematuro e hospitalizado em uma UTI Neonatal é um momento de crise para a família envolvida nessa situação, em especial para a mãe em seu pós-parto. Esses momentos dificeis demandam uma adaptação para que ocorra a superação ou mesmo aceitação dessa condição, requerendo ações por parte daqueles que estão inseridos nesse contexto. $\mathrm{O}$ enfermeiro é um dos profissionais que compõe essa situação, precisando estar capacitado para auxiliar essa mãe neste momento de crise.

A utilização neste estudo, do Sistema Conceitual proposto por KING (1981), mediante a utilização dos conceitos de comunicação e interação, possibilitou trabalhar com as puérperas, considerando-as como um ser que é parte de um sistema semi-aberto e organizado que se denomina família e, que sofre modificações no Sistema Interpessoal propiciadas pela hospitalização de seu filho, como é percebido mediante a identificação dos diagnósticos de Conflito de 
Desempenho dos Papéis dos Pais, Risco para Amamentação Ineficaz, Medo e Risco para Alteração no Desenvolvimento do Apego entre Pais e Filho. Estes diagnósticos envolvem os conceitos propostos no Sistema Conceitual de King, como o de papel, ou seja, a puérpera apresentou um conflito entre o papel de ser mãe, dona-de-casa e esposa, bem como a vivência de ser mãe a distância, devido a separação de seu filho. Portanto, uma versão diferente da expectativa do que é ser mãe.

O diagnóstico de medo presente neste estudo envolveu o conceito de estresse proposto por KING (1981), em que são considerados os sentimentos de apreensão e as situações inoportunas que o indivíduo pode vivenciar que geram um comportamento de estresse.

Acredita-se que a identificação dos diagnósticos de enfermagem apresentados nesse período, tanto fisiológicos como psicológicos, levam ao direcionamento do cuidado, planejado individualmente para atingir metas que, de acordo com KING (1981), devem ser propostas mutuamente.

\section{NURSING DIAGNOSES FROM NANDA'S TAXONOMY IN WOMEN WITH A HOSPITALIZED PRETERM CHILD AND KING'S CONCEPTUAL SYSTEM}

This study aimed at identifying nursing diagnoses presented by mothers with a hospitalized preterm child in an NICU by the use of King's Conceptual System and Taxonomy I of NANDA's nursing diagnoses. The case-study methodology was applied to 35 puerperal women. Eight diagnoses were identified, the frequency of which was over 50\%, as follows: Risk of Infection, Risk of Injury, Impaired Tissue Integrity, Altered Health Maintenance, Risk of Inefficient Breast-feeding; Risk of Altered Parent/Infant Attachment; Fear; Parental Role Conflict and Impaired Social Interaction.

KEY WORDS: nursing diagnosis, preterm newborn, puerperium

\section{LOS DIAGNOSTICOS DE ENFERMERÍA DE LA TAXONOMÍA DE LA NANDA DE MUJERES CON HIJO PREMATURO HOSPITALIZADO Y EL SISTEMA CONCEPTUAL DE KING}

Los objetivos de este estudio fueron: identificar los diagnósticos de enfermería de madres con hijo prematuro hospitalizado en una Unidad de Tratamiento Intensivo Neonatal mediante la utilización del Sistema Conceptual de King y la Taxonomía I de los diagnósticos de enfermeria de la NANDA (1999). Para esto se utilizó la metodología del estudio de caso a 35 puerperas. Fueron identificados ocho diagnósticos con frecuencia igual o mayor de 50\%, el Riesgo para Daño, Riesgo para Lesión, Integridad del Tejido Dañada, Mantenimiento de la Salud Alterada, Riesgo para lactancia Ineficaz; Riesgo para Alteración en el Desarrollo del Apego entre Padres e Hijo; Miedo; Conflicto en el desempeño del Papel Paternal.

TÉRMINOS CLAVES: diagnóstico de enfermería, recién nacido prematuro, puerpera

\section{REFERÊNCIAS BIBLIOGRÁFICAS}

01. ANGELO, M. Experiência das mães na visita ao filho hospitalizado. São Paulo, 1982. 70p. Dissertação (Mestrado) - Escola de Enfermagem, Universidade de São Paulo.

02. ANGELO, M. Com a família em tempos difíceis. São Paulo, 1997. 117p. Tese (Livre Docência) Escola de Enfermagem, Universidade de São Paulo.

03. BARBOSA, V. L. Conhecimento e opiniões de enfermeiras sobre a participação da mãe na assistência ao RN prematuro. São Paulo, 1990. 129p. Dissertação (Mestrado) - Escola de Enfermagem, Universidade Federal de São Paulo.

04. BEZERRA, L. de F.R.; FRAGA, M. de N.de O. Acompanhar um filho hospitalizado: compreendendo a vivência da mãe. Rev. Bras. Enfermagem, Brasília, v. 49, n. 4, p. 611-24, out./dez. 1996.
05. BOECHS, A. E. Famílias vivenciando a chegada de um recém-nascido. Rev. Bras. Enfermagem, Brasília, v. 45, n. 2/3, p. 165-71, abril/set. 1992.

06. BOWLBY, J. Cuidados maternos e saúde mental. (Maternal Care \& Mental Health). São Paulo: Martins Fontes, 1981.

07. BRAZELTON, T. B. O desenvolvimento do apego: uma família em formação. (On becoming a family - The growth of attachment). Porto Alegre: Artes Médicas, 1988.

08. CARPENITO, L.J. Manual de diagnósticos de enfermagem. (Handbook of nursing diagnosis) Porto Alegre: Artes Médicas, 1999.

9. CARVALHO, E.C.de; JESUS, C.A.C. Raciocínio clínico na formulação do diagnóstico de enfermagem para o indivíduo. In: ANTUNES, M. J.; SILVA, M.T. N. (org.). O uso do diagnóstico na prática de enfermagem. Brasília: ABEn, 1997. p. 27-38. 
10. chu ly, t. s.w. Behavior change of a primipara with cesarean section during early postpartum period. Hu Li Tsa Chih, Tailândia, v. 44 n. 3, p. 25-31, Jun. 1997.(abstract)

11. GARCIA, T.R.; CARVALHO, E.C.; PELÁ, N.T.R. Operacionalização do modelo de King: aplicabilidade a gestantes solteiras. In: CONGRESSO BRASILEIRODEENFERMAGEM, 45, Olinda-PE, 1993. Programas e Resumos. Olinda: ABEnNacional/ABEn-PE, 1993. p. 18.

12. GEORGE, J.B. Nursing theories: the base for professional practice. 3. ed. Notwalk, Connecticut: Appleton \& Lange, 1990. Cap. 13.

13. GOMES, M. M. F. Ter o filho na Unidade de Terapia Intensiva Neonatal: o significado para os pais. São Paulo, 1992. 112p. Dissertação (Mestrado) - Escola de Enfermagem, Universidade Federal de São Paulo.

14. HAMELIN, K.; SAYDAK, M.; BRAMADAT, I. A. Interviewing mothers of high-risk infants: what are their support needs? Can. Nurse, v. 93, n. 6, p. 3538, jun. 1997.

15. KIMURA, A.F. O nascimento: os familiares como participantes do processo de cuidar do recém-nascido Acta Paul. Enfermagem, São Paulo, v. 9, p. 45-47, 1996. Número especial.

16. KING, I.M A theory for nursing: systems, concepts, process. New York: John Wiley, 1981.

17. KLAUS, M.H.; KENNEL, J.H. Pais/bebê: a formação do apego. (Parent-Infant Bonding). Porto Alegre: Artes Médicas, 1993.

18. LÜDKE, M.; ANDRÉ, M.E.D.A. Pesquisa em educação: abordagens qualitativas. São Paulo: EPU, 1986.

19. MARQUES, C.L.B.; ELSEN, I. Aplicação de uma teoria à prática de enfermagem: um relato de experiência. In: BUB, L.I.R.; PENNA, C. M. de M.; ATHOLF, C.R.; ELSEN, I.; PATRÍCIO, Z. M. Marcos para a prática de enfermagem com famílias. Florianópolis: Editora da UFSC, 1994. p.161-177.

20. MILES, M. S.; FUNK, S. G.; KASPER, M. A. The stress response of mothers and fathers of preterm infants.

Res. Nurs. Health, v. 15, p. 261-269, 1992.

21. MOTTA, M. da G.C. A doença da criança redefinindo o universo familiar. In: COLÓQUIO PANAMERICANO DE ENFERMAGEM, 6, Ribeirão Preto, 1998. Livro de Resumos. Ribeirão Preto, 1998. p. 332.
22.NORTH AMERICAN NURSING DIAGNOSES ASSOCIATION - NANDA - Nursing diagnoses: definitions \& classification - 1999 - 2000. Philadelphia: North American Nursing Diagnosis Association, 1999.

23. POLIT, D. F.; HUNGLER, B. P. Fundamentos de pesquisa em enfermagem (Essentials of Nursing research - methods, appraisal, and utilization) 3. ed. Porto Alegre: Artes Médicas, 1995.

24. REEDER, S.J. ; MARTIN, L.L. ; KONIAK-GRIEFFIN, D. Maternity nursing: family, newborn and women's heatlh care. 8. ed. Philadelphia: Linpincott, 1997. Cap. 25-27, 37, 38.

25. RISNER, P.B. Diagnosis: analysis and sinthesis of data In: CHRISTENSEN, P.J.; KENNEY, J.W. Nursing process: conceptual models. 4. ed. St. Louis: Mosby, 1990. p 124-150.

26. SCOCHI, C. G. S.; LIMA, R. A. G.; DELÁCIO, L. B.; MORAIS, G. R. de O conhecimento de puérperas sobre o cuidado com o recém-nascido: avaliação da influência da internação em uma maternidade de um hospital-escola. Rev. Bras. Enfermagem, Brasília, v. 45, n. 1, p. 36-43, jan./mar. 1992.

27. STEVENS, K. A. Nursing diagnoses in wellness childbearing settings. JOGNN, v. 17, n.5, p. 329-36, Sept/Oct. 1988.

28. SOUZA, A. B. G. Buscando uma chance para o filho vir a ser: a experiência do pai na unidade de terapia intensiva. São Paulo, 1997. 100 p. Dissertação (Mestrado) - Escola de Enfermagem de Ribeirão Preto, Universidade de São Paulo.

29. TRIBOTTI, S.; LYONS, N.; BLACKBURN, S.; STEIN, M.; WITHERS, J. Nursing diagnoses for the postpartum woman. JOGNN, v. 17, n. 6, p. 410-16, Nov./Dec. 1988.

30. VALE, I.N. Risco para amamentação ineficaz: proposta de diagnóstico de enfermagem. São Paulo, 1999. 109p. Tese (Doutorado) - Escola de enfermagem, Universidade de São Paulo.

31. WILLIANS, L.R.; COOPER, M.K. Nurse-managed postpartum home care. JOGNN, v. 22, n. 1, p. 25-31, Jan./Fev. 1993.

32. ZAVASCHI, M.L.S.; OLIVEIRA, V.Z. de; SILVEIRA, M.D.T. da Aspectos emocionais de mães de recémnascidos hospitalizados na Unidade de Neotatologia do Hospital de Clínicas de Porto Alegre. Rev. HCPA \& Fac. Med. Univ. Fed. Rio Gd. do Sul, v. 5, n. 1, p. 15-19, jun.1985. 